\title{
Black Holes in the Universe: Generalized Lemaître-Tolman-Bondi Solutions
}

\author{
Changjun $\mathrm{Gad}^{*}$ and Xuelei Chen \\ The National Astronomical Observatories, Chinese Academy of Sciences, Beijing, 100012, China \\ You-Gen Shen甲 \\ Shanghai Astronomical Observatory, Chinese Academy of Sciences, Shanghai 200030, China and \\ Joint Institute for Galaxy and Cosmology of SHAO and USTC, Shanghai 200030, China \\ Valerio Faraon: \\ Physics Department, Bishop's University, 2600 College Street, Sherbrooke, Québec, Canada J1M 1Z77
}

(Dated: October 19, 2018)

\begin{abstract}
We present new exact solutions which presumably describe black holes in the background of a spatially flat, pressureless dark matter (DM)-, or dark matter plus dark energy (DM+DE)-, or quintom-dominated universe. These solutions generalize Lemaître-Tolman-Bondi metrics. For a DM- or (DM+DE)-dominated universe, the area of the black hole apparent horizon (AH) decreases with the expansion of the universe while that of the cosmic AH increases. However, for a quintomdominated universe, the black hole $\mathrm{AH}$ first shrinks and then expands, while the cosmic $\mathrm{AH}$ first expands and then shrinks. A (DM+DE)-dominated universe containing a black hole will evolve to the Schwarzschild-de Sitter solution with both AHs approaching constant size. In a quintomdominated universe, the black hole and cosmic AHs will coincide at a certain time, after which the singularity becomes naked, violating Cosmic Censorship.
\end{abstract}

\section{INTRODUCTION}

What is the relation between cosmic expansion and local physics? Do local gravitational system, i.e., stars, galaxies, galaxy clusters, or even black holes, expand with the cosmic expansion? This issue has received much attention over the years [1 21], however, these discussions often produced contradictory results leaving the issue rather ambiguous (see [23 25] for reviews). It is generally believed that these contradictions are due to the use of different or unphysical coordinates, or of unphysical solutions [9].

In order to address this issue, McVittie introduced his renowned solution [26] in 1933; this was intended as describing a point mass embedded in a FriedmannRobertson-Walker (FRW) universe, but modern studies revealed that the McVittie metric cannot describe a point mass [2, 6, 11, 27], but is rather interpreted as describing a black hole in a cosmological background [46, 47]. Einstein and Strauss [28] introduced the Swiss-cheese model which is, however, unable to describe the Solar System and suffers from other limitations [1, 23]. Next, the Vaidya [29], Thakurta, Sultana-Dyer [15, 30], and Faraoni-Jacques [31] solutions were found, and these solutions also describe black holes embedded in FRW universes, each with some restrictions. Either the cosmic fluid is restricted to be a cosmological constant (yielding the Schwarzschild-de Sitter solution, a very special case,

\footnotetext{
*Electronic address: gaocj@bao.ac.cn

${ }^{\dagger}$ Electronic address: $x$ xuelei@cosmology.bao.ac.cn

‡Electronic address: ygshen@center.shao.ac.cn

$\S$ Electronic address: vfaraoni@ubishops.ca
}

indeed), or it must be a mixture of two perfect fluids, one of which is a null dust (for the Sultana-Dyer solution), or it is an imperfect fluid with heat flow instead of a simple perfect fluid (for the Faraoni-Jacques solution) [21, 27]. It is of interest to look for simpler exact solutions with a single perfect fluid as a source to address the issue of cosmic expansion versus local physics. The purpose of the present paper is to derive such new solutions representing a black hole in a FRW universe with a cosmic fluid consisting of dark matter, or of dark matter plus dark energy (possibly a cosmological constant), or of quintom matter 32]. These types of solution are also useful, if nothing else as toy models, in the study of dynamical horizons and their thermodynamics 22, 42 45] and of primordial black holes [33] as probes of the early universe [34, 35].

Regarding the relation between cosmic expansion and local physics, Noerdlinger and Petrosian conclude their work [4] by stating: "Consider the possible expansion of clusters or superclusters of galaxies, of mean rest-mass density $\rho_{c}$, immersed in a universe containing a gas of particles having energy density $\rho$. It is shown that when $\rho>\rho_{c}$, the clusters or superclusters expand with the universe, but if $\rho_{c} \gg \rho$, the expansion is reduced in the ratio $\rho / \rho_{c}$ ". Unfortunately, this conclusion is drawn from the McVittie solution, which can not describe realistically a point mass, a star, or a cluster of galaxies in a FRW background. A similar conclusion was reached by Price [18] who discovered the "all or nothing" behavior (i.e., weakly coupled systems are comoving while strongly coupled ones resist the cosmic expansion). However, Price's result applies only to a de Sitter background, not to a general FRW universe. As is well-known, de Sitter space is very special: the de Sitter metric can be put in static form in the spacetime region between the black hole and the cosmological horizons, which explains why strongly 
bound systems in this background do not expand [31].

In this paper, we present new solutions which we propose to interpret as describing cosmological black holes embedded in FRW backgrounds, with the advantage over previous solutions [15, 26, 29 31] that they are sourced by a single perfect fluid, without heat flux. These solutions lead to surprising results: the black hole $\mathrm{AH}$ decreases with the expansion of the universe when the latter is DM- or (DM+DE)-dominated. Since the Misner-Sharp mass is a half of the radius of the $\mathrm{AH}$, also the black hole mass decreases with the cosmic expansion. As the most strongly bound gravitational system, it is intuitive that the size of a black hole should increase with the expansion of the universe due to the swallowing of surrounding cosmic matter. However, this picture is incorrect in general as the cosmic expansion "wins" over the local gravitational attraction of the black hole. The universe is always expanding after the Big Bang and the energy density of the cosmic fluid decreases with cosmic time. The seemingly bizarre behavior of the Misner-Sharp mass derives from the fact that it is really a mass sum (i.e., the mass of the background fluid is included), and coincides with the Hawking-Hayward quasi-local mass [21]. We note that this is a purely classical effect which makes the black hole mass decrease and has nothing to do with Hawking radiation 36].

In a quintom-dominated universe, during the matter era the black hole mass decreases, while during the phantom-dominated epoch it increases. The physical reason is that the cosmic density first decreases and then increases. Since the Misner-Sharp (MS) mass is the sum of the black hole mass and of the background mass, the black hole mass first decreases and then increases. One interesting result is that the black hole singularity will become naked before the Big Rip occurs, which violates the Cosmic Censorship Conjecture [37]. So if the latter is correct, phantom matter may not exist in nature.

In this paper, we use units in which the speed of light $c$ and Newton's constant $G$ assume the value unity, and the signature +2 for the metric. The paper is organized as follows: first we show that several known solutions can not describe a black hole embedded in a matterdominated universe. Second, we present new solutions describing a black hole in a matter-dominated universe, a dark matter plus cosmological constant-dominated universe, and a quintom-dominated universe. The last section contains the conclusions.

\section{THE MCVITTIE SOLUTION}

A natural starting point to investigate the relation between cosmic expansion and local physics is the 1933 McVittie solution [26], which can be written in isotropic coordinates as

$$
\begin{aligned}
d s^{2}= & -\frac{\left[1-\frac{M_{0}}{2 a(t) r}\right]^{2}}{\left[1+\frac{M_{0}}{2 a(t) r}\right]^{2}} d t^{2}+a(t)^{2}\left[1+\frac{M_{0}}{2 a(t) r}\right]^{4} \\
& \cdot\left(d r^{2}+r^{2} d \Omega^{2}\right)
\end{aligned}
$$

where the constant $M_{0}$ reduces to the physical black hole mass when $a(t)=$ const. (this can be seen by rescaling the radial coordinate $r \rightarrow \tilde{r} \equiv a r$ ), and the scale factor $a(t)$ is an arbitrary function of the cosmic time $t$. When $M_{0}=0$, the solution reduces to the spatially flat FRW metric, and at a first glance, it can be understood as representing a Schwarzschild black hole embedded in a spatially flat FRW universe [4]. However, it is now known that, with the exception of the Schwarzschild-de Sitter solution, matters are more complicated because the McVittie metric is singular on the 2-sphere $r=2 M_{0} a$ (which reduces to the Schwarzschild horizon if $a=$ const.) [6] and this singularity is spacelike [11]. It was claimed in the past that the McVittie metric describes a point mass located at $r=0$ and embedded in a FRW universe. However, this point mass is, in general, surrounded by the singularity at $r=M_{0} /(2 a)$. This singularity was studied in Ref. [6]. Nolan [2] showed that it is a weak singularity in the sense that an object falling onto the $r=M_{0} /(2 a)$ surface is not shrunk to zero volume, and therefore the energy density of the surrounding fluid is finite. However, the pressure diverges at $r=M_{0} /(2 a)$ together with the Ricci scalar $R$ [6, 27]. While there is little doubt that the McVittie metric represents some kind of strongly gravitating central object, its physical interpretation is not completely clear and is still a subject of debate [6, 11, 46, 47].

In view of this situation we show that, with the exception of the Schwarzschild-de Sitter solution, the McVittie solution is unable to describe a black hole or a point mass in a FRW universe when the equation of state of the cosmic perfect fluid is $p=w(t) \rho$. The proof of this statement proceeds as follows. The McVittie solution was obtained by forbidding explicitly the accretion of cosmic fluid onto the central object. This requirement corresponds to $G_{0}^{1}=0$, which in turn implies that the stress-energy tensor component $T_{0}^{1}=0$ and there is no radial flow of energy. Let us assume that the energymomentum tensor that sources the McVittie metric is that of a perfect fluid, $T_{\mu \nu}=(\rho+p) U_{\mu} U_{\nu}+p g_{\mu \nu}$, where $U_{\mu}$ is the 4-velocity of the comoving observer, and $\rho$ and $p$ are the density and pressure of the fluid, respectively.

By substituting the metric and the energy-momentum tensor into the Einstein equations $G_{\mu \nu}=8 \pi T_{\mu \nu}$, the resulting Einstein equations can be arranged as

$$
3 H^{2}=8 \pi \rho,
$$

$$
\frac{2 \ddot{a}}{a}+H^{2}=-8 \pi p-\frac{M_{0}}{a r}\left(\dot{H}-\frac{3}{2} H^{2}-4 \pi p\right),
$$


where $H=\dot{a} / a$ is the Hubble parameter and an overdot denotes differentiation with respect to the cosmic time $t$. We recognize eqs. (2) and (3) as the Friedmann and the acceleration equations, respectively.

We have $p=0$ and $p=\rho / 3$ for the matter-dominated and the radiation-dominated eras, respectively, while $p=w(t) \rho$ with $w<-1 / 3$ for a dark energy-dominated universe. For all these cases, the equation of state assumes the form $p=w(t) \rho$. Eq. (2) tells us that $\rho$ depends only on $t$ and, since $p=w(t) \rho$, the same is true for $p$. In eq. (3), the expressions $\frac{2 \ddot{a}}{a}+H^{2}$ and $-8 \pi p$ depend only on $t$, while $-\frac{M_{0}}{a r}\left(\dot{H}-\frac{3}{2} H^{2}-4 \pi p\right)$ depends on both $t$ and $r$, therefore eq. (3) is satisfied if and only if

$$
\begin{aligned}
& 3 H^{2}=8 \pi \rho, \quad \frac{2 \ddot{a}}{a}+H^{2}+8 \pi p=0, \\
& \dot{H}-\frac{3}{2} H^{2}-4 \pi p=0 .
\end{aligned}
$$

These admit the unique solution

$$
H=\text { const. }, \quad p=-\rho=\text { const. }, \quad a(t)=\mathrm{e}^{H t},
$$

which is the Schwarzschild-de Sitter spacetime. Therefore, the McVittie solution can not describe a black hole embedded in a matter-dominated, radiation-dominated, or dark energy-dominated universe with perfect fluid equation of state $p=w(t) \rho$. In one word, given the equation of state $p=w(t) \rho$, the McVittie solution is nothing but the Schwarzschild-de Sitter solution.

Actually, Kaloper et al. [46] have argued that in the presence of a cosmological constant, McVittie's solution does in fact represent a black hole in an expanding universe. Here we provide independent support for this statement.

Recently, assuming a specific function $H(t)$ in the McVittie solution, Lake and Abdelqader [47] performed a detailed study of the particular McVittie solution and corroborated the conclusion of Kaloper et al. Moreover, using a tetrad-based method for solving Einstein's field equations, Nandra et al. [48] obtained solutions describing objects embedded in FRW universes which provide a new perspective on the McVittie metric. The study of the global structure of the McVittie solution in [47] revealed unexpected features which make this solution much more complicated than it would seem at a first sight. Something similar probably happens for the new solutions presented here, although we will leave the detailed investigation of radial null geodesics and of the global structure to future work.

\section{THE FARAONI-JACQUES SOLUTION}

Because the McVittie solution can not describe a black hole in a FRW universe, we turn our attention to the Faraoni-Jacques solution [31], which is a generalization of the McVittie solution

$$
\begin{aligned}
d s^{2}= & -\frac{\left[1-\frac{M(t)}{2 a(t) r}\right]^{2}}{\left[1+\frac{M(t)}{2 a(t) r}\right]^{2}} d t^{2}+a(t)^{2}\left[1+\frac{M(t)}{2 a(t) r}\right]^{4} \\
& \cdot\left(d r^{2}+r^{2} d \Omega^{2}\right)
\end{aligned}
$$

in which the function $M(t)$ replaces the constant $M_{0}$ of the McVittie metric. This corresponds to lifting the McVittie non-accretion restriction $G_{0}^{1}=0$. This solution seems to describe a cosmological black hole embedded in a FRW background and presents advantages over the previous Thakurta and Sultana-Dyer solutions [15, 30],

$$
\begin{aligned}
d s^{2}= & -\frac{\left(1-\frac{M_{0}}{2 r}\right)^{2}}{\left(1+\frac{M_{0}}{2 r}\right)^{2}} d t^{2}+a(t)^{2}\left(1+\frac{M_{0}}{2 r}\right)^{4} \\
& \cdot\left(d r^{2}+r^{2} d \Omega^{2}\right)
\end{aligned}
$$

in the sense that both the energy density and the pressure are finite near the black hole horizon, and the energy density is positive-definite [31]. Now let us examine whether it can describe a black hole embedded in FRW universe sourced by a perfect fluid.

We assume that the cosmological matter is described by the single perfect fluid energy-momentum tensor

$$
T_{\mu \nu}=(\rho+p) U_{\mu} U_{\nu}+p g_{\mu \nu},
$$

where $U^{\mu}$ is the 4 -velocity of the fluid. The only nonvanishing components of the Einstein tensor are

$$
\begin{aligned}
& G_{0}^{0} \neq 0, \quad G_{0}^{1} \neq 0, \\
& G_{1}^{1}=G_{2}^{2}=G_{3}^{3} \neq 0 .
\end{aligned}
$$

If a radial energy flow is allowed, the fluid four-velocity is

$$
U^{\mu}=\left(U^{0}, U^{1}, 0,0\right),
$$

and the normalization $U_{\mu} U^{\mu}=-1$ gives

$$
U^{\mu}=\left[\sqrt{-g^{00}-a^{2} v^{2} g^{00}\left(1+\frac{M(t)}{2 a(t) r}\right)^{4}}, v, 0,0\right],
$$

where $v$ is the proper 3-velocity of the fluid. From $G_{11}=$ $G_{22}$ we conclude that

$$
(\rho+p) a^{2} v^{2}\left(1+\frac{M(t)}{2 a(t) r}\right)^{4}=0 .
$$

We have two types of solutions characterized by

$$
v=0, \quad M=M_{0}
$$

(which is the McVittie solution), or

$$
p=-\rho,
$$


which gives the Schwarzschild-de Sitter solution. Therefore, the Faraoni-Jacques solution (and also the Thakurta and Sultana-Dyer solutions) can not describe a black hole embedded in a matter-dominated universe. In general, they can not describe a black hole embedded in a single perfect fluid-dominated universe. However, the FaraoniJacques solution can describe a black hole embedded in FRW Universe provided that the source is given by an imperfect fluid with a heat flux:

$$
T_{\mu \nu}=(p+\rho) U_{\mu} U_{\nu}+p g_{\mu \nu}+q_{\mu} U_{\nu}+q_{\nu} U_{\mu}
$$

where $U^{\mu}=\left(\sqrt{-g^{00}}, 0,0,0\right)$ and $q^{\mu}=(0, q, 0,0)$, where $q$ is the heat flux density. This has been shown by us in [21].

\section{THE VAIDYA SOLUTION}

The Vaidya metric is obtained by conformally transforming the Minkowski one:

$$
\begin{aligned}
d s^{2}= & a^{2}(\eta)\left(-d \eta^{2}+d r^{2}+r^{2} d \theta^{2}+r^{2} \sin ^{2} \theta d \phi^{2}\right) \\
& +\frac{2 M_{0}}{r}(d \eta+d r)^{2}
\end{aligned}
$$

where $M_{0}$ is the black hole mass and $\eta$ is the conformal time. When $M_{0}=0$ and $a=\eta^{2}$, this metric reduces to that for a matter-dominated universe. On the other hand, when $a=$ const., it reduces to the Schwarzschild metric. At a first glance, one is tricked again into believing that this metric may describe a black hole in a matter-dominated universe but this is incorrect, as shown below. is

The energy-momentum tensor of dust or dark matter

$$
T_{\mu \nu}=\rho_{d} U_{\mu} U_{\nu}
$$

Due to spherical symmetry, the four-velocity $U^{\mu}$ is written as eq. (10). We have two vanishing components of $T_{\mu \nu}$, i.e., $T_{2}^{2}$ and $T_{3}^{3}$, hence $G_{2}^{2}=G_{3}^{3}=0$. Given the metric (16), $G_{2}^{2}=G_{3}^{3}$ is calculated as [27],

$$
\left(\frac{2 \ddot{a}}{a^{3}}-\frac{\dot{a}^{2}}{a^{4}}\right)+\left(\frac{2 m \ddot{a}}{r a^{5}}-\frac{4 m \dot{a}^{2}}{r a^{6}}\right)=0 .
$$

The terms in the first bracket depend only on time, while those in the second bracket are $r$-dependent, so eq. (18) is satisfied if and only if

$$
\frac{2 \ddot{a}}{a^{3}}-\frac{\dot{a}^{2}}{a^{4}}=0, \quad \frac{\ddot{a}}{a^{5}}-\frac{2 \dot{a}^{2}}{a^{6}}=0 .
$$

Then we necessarily have the trivial solution $a=$ const., which is just the Schwarzschild metric. This shows that the Vaidya solution can not describe a black hole embedded in a matter-dominated universe, either.

Thus far, we have shown that several known exact solutions can not describe a black hole in a matter-dominated universe. In the next section, we look for such a solution.

\section{BLACK HOLE IN A MATTER UNIVERSE}

A spherically symmetric inhomogeneous gravitational field produced by dark matter with energymomentum (17) is described by the Lemaitre-TolmanBondi (LTB) metric [38] which, in the notation of [39], is given by

$$
d s^{2}=-d t^{2}+\frac{R^{\prime 2}}{1+f} d r^{2}+R^{2} d \Omega^{2} .
$$

Here $f$ is an arbitrary function of the comoving coordinate $r$ satisfying $f>1, R(t, r)$ is the physical radius at time $t$ and coordinate radius $r$, while a prime represents differentiation with respect to $r$.

With the energy momentum tensor (17) and the metric (20), the Einstein equations read

$$
\begin{gathered}
\frac{3\left(\dot{R}^{2}-f\right)}{R^{2}}=\frac{8 \pi F}{R^{3}}, \\
\frac{F^{\prime}}{R^{2} R^{\prime}}=3 \rho_{d},
\end{gathered}
$$

where an overdot and a prime represent partial differentiation with respect to $t$ and $r$, respectively, and $F(r)$ is an arbitrary function of $r$. If we substitute

$$
f=-f_{0} r^{2}, \quad F=\rho_{0} r^{3}, \quad R=a(t) r
$$

into eqs. (21) and (22), the latter become

$$
\frac{3\left(\dot{a}^{2}+f_{0}\right)}{a^{2}}=\frac{8 \pi \rho_{0}}{a^{3}}, \quad \rho_{d}=\frac{\rho_{0}}{a^{3}},
$$

with $f_{0}$ and $\rho_{0}$ constants. We recognize eq. (24) as the Friedmann equation with a dust source, hence $f_{0}$ represents the spatial curvature and $\rho_{0}$ the density of the universe at time $a_{0}=1$. The observed universe is spatially flat with good accuracy (probably due to inflation) and we can neglect the curvature term in what follows by setting $f=0$.

For an arbitrary function $F(r)$ and $f=0$, the solution of eq. (21) was given by Tolman [40] and Oppenheimer and Snyder [41] in their pioneering investigations of the gravitational collapse of dust as

$$
R=\left[h(r)+\frac{3}{2} \sqrt{\frac{8 \pi}{3} F t}\right]^{2 / 3},
$$

where the integration "constant" $h$ is an arbitrary function of $r$. Substitution of eq. (25) into (20) yields

$$
d s^{2}=-d t^{2}+\frac{1}{R}(d h+\sqrt{6 \pi} t d(\sqrt{F}))^{2}+R^{2} d \Omega^{2} .
$$

There is actually less freedom in eq. (25) than is apparent from the two arbitrary functions $F$ and $h$ since $F$ can be taken as a new radial variable. It is convenient to choose

$$
h=r^{3 / 2}
$$


and

$$
R=\left[r^{3 / 2}+\frac{3}{2} \sqrt{\frac{8 \pi}{3} F} t\right]^{2 / 3} .
$$

Eq. (28) is the general solution representing a spherically symmetric gravitational field with dust as a source. As shown above, this can describe a dust-dominated universe. On the other hand, if the dust disappears, it describes a vacuum, spherically symmetric, gravitational field and reduces to the Schwarzschild metric due to the Jebsen-Birkhoff theorem. By combining these considerations, one guesses that eq. (28) may describe a Schwarzschild black hole immersed in a dust-dominated universe. In the following, this claim is examined.

\section{A. Minkowski space}

By solving the Einstein equations with $\rho_{d}=0$, one finds the vacuum solution

$$
F=F_{0},
$$

where $F_{0}$ is an integration constant. If $F_{0}=0$, we obtain $R=r$ and we recover the Minkowski solution.

\section{B. The Schwarzschild solution}

If $F_{0} \neq 0$, we recover the Schwarzschild solution. It is convenient to set $F=\frac{3 F_{0}^{2}}{8 \pi}$ with $F_{0}$ a positive constant. Then, the Schwarzschild solution is given by

$$
R=\left[r^{3 / 2}+\frac{3}{2} F_{0} t\right]^{2 / 3},
$$

$F_{0}$ is determined by the mass $m$ of the black hole. In order to show this, let us rewrite the metric in Schwarzschild coordinates, setting

$$
x(t, r)=\left[r^{3 / 2}+\frac{3}{2} F_{0} t\right]^{2 / 3} .
$$

Then, eq. (20) becomes

$$
d s^{2}=-\left(1-\frac{F_{0}^{2}}{x}\right) d t^{2}+d x^{2}-\frac{2 F_{0}}{\sqrt{x}} d t d x+x^{2} d \Omega^{2} .
$$

Using the new time variable

$$
T=t-t_{0}+2 F_{0} \sqrt{x}+F_{0}^{2} \ln \left(\frac{\sqrt{x}-F_{0}}{\sqrt{x}+F_{0}}\right),
$$

one obtains

$$
d s^{2}=-\left(1-\frac{F_{0}^{2}}{x}\right) d T^{2}+\left(1-\frac{F_{0}^{2}}{x}\right)^{-1} d x^{2}+x^{2} d \Omega^{2},
$$

i.e., the Schwarzschild solution. The physical meaning of $F_{0}$ is derived from $F_{0}=\sqrt{2 m}$ and the Schwarzschild solution is given by

$$
R=\left[r^{3 / 2}+\frac{3}{2} \sqrt{2 m} t\right]^{2 / 3} .
$$

\section{Dust-dominated universe}

Eqs. (22) and (28) show that the homogeneous and isotropic, spatially flat, dust-dominated FRW solution is recovered if and only if $F=\rho_{0} r^{3}$. The dust universe solution is then given by

$$
R=\left[r^{3 / 2}+\frac{3}{2} \sqrt{\frac{8 \pi \rho_{0}}{3}} r^{3 / 2} t\right]^{2 / 3} .
$$

The scale factor and the density are

$$
a=\left[\sqrt{6 \pi \rho_{0}} t+1\right]^{2 / 3}
$$

and

$$
\rho_{d}=\frac{\rho_{0}}{a^{3}},
$$

respectively, and it must be $t \geq t_{0}$, with $t_{0}=-\frac{1}{\sqrt{6 \pi \rho_{0}}}$, representing the Big Bang.

\section{Black hole in a dust-dominated universe}

By combining the considerations above, one expects the metric for a black hole immersed in a dust universe to be given by

$$
R=\left[r^{3 / 2}+\frac{3}{2} \sqrt{2 m} t+\frac{3}{2} \sqrt{\frac{8 \pi \rho_{0}}{3}} r^{3 / 2} t\right]^{2 / 3} .
$$

If $m=0$, this line element describes a dust universe, while if $\rho_{0}=0$, it reduces to a Schwarzschild solution; (39) should describe a black hole immersed in dust universe. It is clear, by comparison of eqs. (39) and (25), that this is a solution of the full Einstein equations. The substitution of eq. (39) into eqs. (21) and (22) gives the energy density

$$
\rho_{d}=\frac{\sqrt{\rho_{0}}\left(3 \sqrt{m}+2 \sqrt{3 \pi \rho_{0}} r^{3 / 2}\right)}{2 \sqrt{3 \pi}\left(\sqrt{6 \pi \rho_{0}} t+1\right) R^{3 / 2}} .
$$

The physical radius $R$ cannot be negative, hence we require that $r \geq 0$ and $t \geq t_{0} \equiv-\sqrt{6 \pi \rho_{0}}$. Then, the density is always positive. If $m=0$, we have $\rho_{d}=\rho_{0} / R^{3}$, i.e., dust or dark matter. Since $R=0$ represents the Big Bang, the latter occurs at the time

$$
t=-\left(\frac{3}{2} \sqrt{2 m} r^{-3 / 2}+\sqrt{6 \pi \rho_{0}}\right)^{-1},
$$

and the energy density is positive everywhere on the spacetime manifold. 


\section{EVOLUTION OF THE APPARENT HORIZONS}

In the Schwarzschild-de Sitter spacetime, there exist a black hole apparent horizon (AH) and a cosmic $\mathrm{AH}$. The metric (20) describes a spherically symmetric and dynamical black hole in a FRW background more general than de Sitter. In this case, the event horizon may not be well-defined, but the $\mathrm{AH}$ always exists. The $\mathrm{AH}$ is a marginally trapped surface with vanishing expansion and has been argued to be a causal horizon for a dynamical spacetime. The $\mathrm{AH}$ is associated with the Hawking temperature, gravitational entropy and other thermodynamical aspects [42 45]. The first law of thermodynamics for the $\mathrm{AH}$ has been derived not only in general relativity but also in several other theories of gravity including the Lovelock, nonlinear, scalar-tensor, and braneworld theories [49 55]. In view of this point, in order to investigate the evolution of the black hole mass, we calculate the energy contained inside the $\mathrm{AH}$ which has been used in black hole thermodynamics in relation with energy flows through the AH [49, 56]. Let us proceed to calculate the radius of the black hole $\mathrm{AH}$.

For a spherically symmetric spacetime with line element $d s^{2}=h_{\mu \nu} d x^{\mu} d x^{\nu}+x^{2} d \Omega^{2}$, the generalized MisnerSharp mass is $M_{M S}=x\left(1-h^{\mu \nu} x_{, \mu} x_{, \nu}\right) / 2$ [57]. At the $\mathrm{AH}$, it is $h^{\mu \nu} x_{, \mu} x_{, \nu}=0$ and the generalized Misner-Sharp mass inside the $\mathrm{AH}$ is simply

$$
M_{M S}=x_{A H} / 2,
$$

where $x_{A H}$ is the radius of the $\mathrm{AH}$. In order to know the evolution of the black hole MS mass, we need to know the $\mathrm{AH}$ radius.

We rewrite eq. (20) in Schwarzschild coordinates using the new spatial coordinate

$$
x=\left[r^{3 / 2}+\frac{3}{2} \sqrt{2 m} t+\frac{3}{2} \sqrt{\frac{8 \pi \rho_{0}}{3}} r^{3 / 2} t\right]^{2 / 3}
$$

instead of $r$, in terms of which eq. (20) is rewritten as

$$
\begin{aligned}
& d s^{2}=-\left[1-\frac{1}{x}\left(\sqrt{2 m}+\sqrt{\frac{8 \rho_{0} \pi}{3}} r^{3 / 2}\right)^{2}\right] d t^{2}+d x^{2} \\
& -\frac{2}{\sqrt{x}}\left(\sqrt{2 m}+\sqrt{\frac{8 \rho_{0} \pi}{3}} r^{3 / 2}\right) d t d x+x^{2} d \Omega^{2}
\end{aligned}
$$

The coordinate system $(t, x, \theta, \phi)$ is not orthogonal but the cross-term $d t d x$ can be eliminated by introducing the new time $T$ defined by

$$
\begin{aligned}
& d T=\frac{1}{J(t, x)}\left\{d t+\frac{1}{\sqrt{x}}\left(\sqrt{2 m}+\sqrt{\frac{8 \rho_{0} \pi}{3}} r^{3 / 2}\right)\right. \\
& \left.\left[1-\frac{1}{x}\left(\sqrt{2 m}+\sqrt{\frac{8 \rho_{0} \pi}{3}} r^{3 / 2}\right)^{2}\right]^{-1} d x\right\},
\end{aligned}
$$

where $J(t, x)$ is an integrating factor which always exists and solves the partial differential equation

$$
\begin{aligned}
\partial_{x} J^{-1}= & \partial_{t}\left\{-J^{-1} \frac{1}{\sqrt{x}}\left(\sqrt{2 m}+\sqrt{\frac{8 \rho_{0} \pi}{3}} r^{3 / 2}\right)\right. \\
& {\left.\left[1-\frac{1}{x}\left(\sqrt{2 m}+\sqrt{\frac{8 \rho_{0} \pi}{3}} r^{3 / 2}\right)^{2}\right]^{-1}\right\} }
\end{aligned}
$$

which guarantees that $d T$ is an exact differential. The metric (44) is cast in the Schwarzschild form

$$
\begin{aligned}
d s^{2}= & -\left[1-\frac{1}{x}\left(\sqrt{2 m}+\sqrt{\frac{8 \rho_{0} \pi}{3}} r^{3 / 2}\right)^{2}\right] J^{2} d T^{2} \\
+ & {\left[1-\frac{1}{x}\left(\sqrt{2 m}+\sqrt{\frac{8 \rho_{0} \pi}{3}} r^{3 / 2}\right)^{2}\right]^{-1} d x^{2} } \\
+ & x^{2} d \Omega^{2}
\end{aligned}
$$

and the equation of the $\mathrm{AHs}$ is

$$
1-\frac{1}{\sqrt{x}}\left(\sqrt{2 m}+\sqrt{\frac{8 \rho_{0} \pi}{3}} r^{3 / 2}\right)=0 .
$$

Substituting eq. (43) into eq. (48), we obtain

$$
2 \sqrt{6 \rho_{0} \pi} x^{3 / 2}-\sqrt{x}\left(3+3 \sqrt{6 \pi \rho_{0}} t\right)+3 \sqrt{2 m}=0 .
$$

This cubic equation for $\sqrt{x}$ has, in general, only two positive roots which represent the black hole $\mathrm{AH}$ and the cosmic $\mathrm{AH}$, respectively. In fact, if $\rho_{0}=0$, we obtain from eq. (49)

$$
x=2 m
$$

the AH of the Schwarzschild black hole. On the other hand, if $m=0$, it is

$$
x=\frac{3}{2}\left(\sqrt{\frac{1}{6 \pi \rho_{0}}}+t\right),
$$

which is the AH of a dust-dominated universe. The black hole and the cosmic AHs are plotted in Fig. 1] As is clear from this figure, the radius of the black hole $\mathrm{AH}$ decreases while that of the cosmic $\mathrm{AH}$ increases as the universe evolves. There was an early time at which the two horizons coincided, and before which both horizons were absent and the singularity was naked. Since the MS mass is proportional to the radius of the black hole $\mathrm{AH}$, the MS mass of a black hole decreases with the evolution of the universe. The reason for this behavior can be understood as follows: with the expansion of the universe, the cosmic density is decreasing, which is equivalent to a decreasing of black hole AH, so the black hole MS mass decreases. 


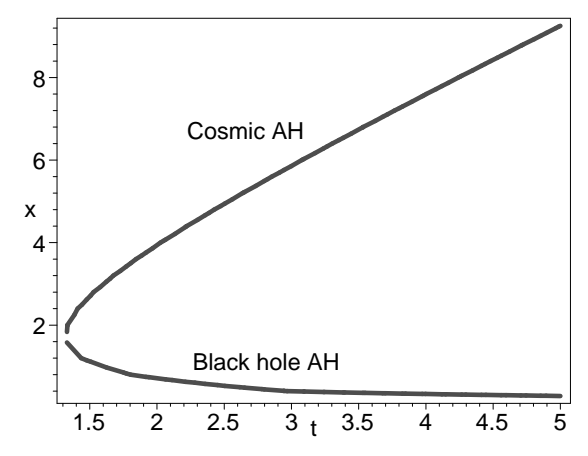

FIG. 1: The size of the black hole AH (lower curve) in a dust-dominated universe decreases with the cosmic expansion while that of the cosmic AH (upper curve) increases. The MS mass of the black hole tends to zero in the future. There was a time at which the two horizons coincided and before which they were absent and the singularity was naked. The plots correspond to the parameter values $m=1, \rho_{0}=0.05$ in Plank units. In the International Units System, they are $m=10^{24} \mathrm{M}_{\odot}$ and $\rho_{0}=0.05 \cdot 10^{-123} \rho_{P}\left(\rho_{P}\right.$ is the Plank density).

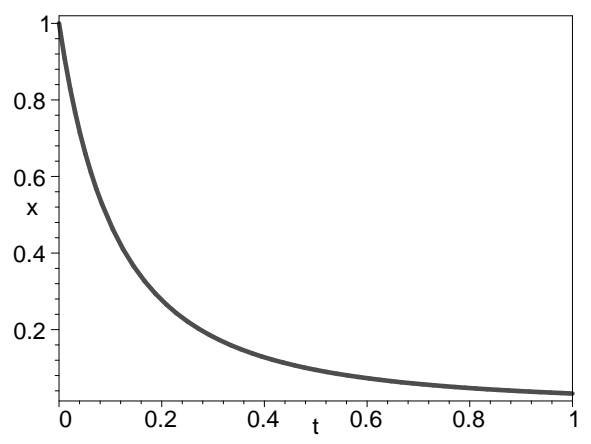

FIG. 2: The $\mathrm{AH}$ of an astronomical black hole in a dustdominated universe decreases with the cosmic expansion. The plots correspond to the parameter value $m=10^{7} \mathrm{M}_{\odot}$. The units of $x$ and $t$ are $2 m$ (the corresponding Schwarzschild radius) and $\mathrm{H}_{0}^{-1}$, respectively.

We note that Fig. 1 is plotted in Plank units $G=c=$ $\hbar=1$. Translating into International units and taking into account the present cosmic density $\rho_{0}=10^{-123} \rho_{P}$ (where $\rho_{P}$ is the Plank density), we have a very supermassive black hole $m=10^{24} \mathrm{M}_{\odot}$ for Fig. 1 The corresponding units of $x$ and $t$ are $10^{5} \mathrm{Mpc}$ and the Hubble time $H_{0}^{-1}$, respectively. To our knowledge, such supermassive black holes may not exist in the universe, so in Fig. 2 we also plot the evolution of the AH for an astronomical black hole with mass $m=10^{7} \mathrm{M}_{\odot}$. We have shifted the Big Bang time to $t=0$.

The naked singularity is present before the appearance of the two apparent horizons and cannot arise from regular initial data.

\section{BLACK HOLE IN A $\Lambda$ CDM UNIVERSE}

Astronomical observations show that the present-day universe is dominated by dark energy [58, 59], hence we seek exact solutions describing a black hole immersed in a mixture of dark matter and dark energy. The corresponding stress-energy tensor is

$$
T_{\mu \nu}=\rho_{d} U_{\mu} U_{\nu}+\rho_{\Lambda} g_{\mu \nu} .
$$

The first term on the right hand side describes dark matter and the second term describes dark energy. $\rho_{\Lambda}$ is a positive constant ${ }^{1}$ We assume the line element to be of the form

$$
d s^{2}=-d t^{2}+e^{\bar{\omega}} d r^{2}+e^{\omega} d \Omega^{2},
$$

where $\bar{\omega}$ and $\omega$ are functions of $t$ and $r$. In comoving coordinates, the four-velocity is $U^{\mu}=(1,0,0,0)$ and the Einstein equations are

$$
\begin{aligned}
& G_{0}^{0}=8 \pi\left(\rho_{d}+\rho_{\Lambda}\right), \quad G_{1}^{0}=0, \\
& G_{1}^{1}=8 \pi \rho_{\Lambda}, \quad G_{2}^{2}=8 \pi \rho_{\Lambda} .
\end{aligned}
$$

From $G_{1}^{0}=0$ one obtains

$$
e^{\bar{\omega}}=e^{\omega} \omega^{2} / 4
$$

Note that there is no accretion onto the central object, as in the McVittie metric. Following Oppenheimer and Snyder, we have set the integration "constant" $(f(R)$ in [41]) to unity. Substituting eq. (55) into eq. (54), we obtain

$$
\ddot{\omega}^{\prime}+\frac{3}{2} \dot{\omega} \dot{\omega}^{\prime}=0
$$

Integration with respect to $r$ yields

$$
\ddot{\omega}+\frac{3}{4} \dot{\omega}^{2}=-8 \pi K(t),
$$

where $K$ is an integration "constant" (an arbitrary function of $t$ ). This equation is consistent with $G_{1}^{1}=8 \pi \rho_{\Lambda}$ if and only if

$$
K(t)=-\rho_{\Lambda}
$$

The Einstein equations then reduce to

$$
\begin{gathered}
G_{1}^{1}=\ddot{\omega}+\frac{3}{4} \dot{\omega}^{2}=8 \pi \rho_{\Lambda}, \\
G_{0}^{0}=\frac{\dot{\omega}^{\prime} \dot{\omega}}{\omega^{\prime}}+\frac{3}{4} \dot{\omega}^{2}=8 \pi\left(\rho_{d}+\rho_{\Lambda}\right) .
\end{gathered}
$$

1 There is evidence in [46] and [47] that the presence of a cosmological constant makes a significant difference in the McVittie solution, and we expect that this is the case also for our generalized Lemaître-Tolman-Bondi solutions. 
Using $k \equiv \sqrt{6 \pi \rho_{\Lambda}}$, the Einstein equations have the solution

$$
\begin{aligned}
& e^{\omega}=\left(S e^{k t}+P e^{-k t}\right)^{4 / 3}, \\
& e^{\bar{\omega}}=e^{\omega} \omega^{\prime 2} / 4
\end{aligned}
$$

where $S$ and $P$ are arbitrary functions of $r$. Substitution of eqs. (60) into eq. (53) and the use of $S$ as a new spatial coordinate leave only one degree of freedom. It is convenient to choose

$$
S=r^{3 / 2}
$$

which has the advantage that if $P=0$ and $\rho_{\Lambda}=0$, the line element (53) reduces to the Minkowski one.

\section{A. The Schwarzschild-de Sitter solution}

The Einstein equations with $\rho_{d}=0$ yield

$$
P=S_{0} r^{-3 / 2}
$$

where $S_{0}$ is an integration constant. The Schwarzschildde Sitter solution is given by

$$
\begin{aligned}
e^{\omega} & =\left(r^{3 / 2} e^{k t}+S_{0} r^{-3 / 2} e^{-k t}\right)^{4 / 3}, \\
e^{\bar{\omega}} & =e^{\omega} \omega^{2} / 4 .
\end{aligned}
$$

The physical meaning of $S_{0}$ can be understood after rewriting eq. (63) in Schwarzschild coordinates; setting

$$
x=\left(r^{3 / 2} e^{k t}+S_{0} r^{-3 / 2} e^{-k t}\right)^{2 / 3}
$$

the line element (53) becomes

$$
\begin{aligned}
d s^{2}= & -\left[1-\frac{4 k^{2}\left(x^{3}-4 S_{0}\right)}{9 x}\right] d t^{2}+d x^{2} \\
& -\frac{4 k}{3 \sqrt{x}} \sqrt{x^{3}-4 S_{0}} d t d x+x^{2} d \Omega^{2}
\end{aligned}
$$

Introducing the new time

$$
\begin{aligned}
T= & t+\int\left[1-\frac{4 k^{2}\left(x^{3}-4 S_{0}\right)}{9 x}\right]^{-1} \\
& \frac{2 k}{3 \sqrt{x}} \sqrt{x^{3}-4 S_{0}} d x
\end{aligned}
$$

the metric (65) simplifies to

$$
\begin{aligned}
d s^{2}= & -\left(1+\frac{16 k^{2} S_{0}}{9 x}-\frac{4 k^{2}}{9} x^{2}\right) d T^{2}+x^{2} d \Omega^{2} \\
& +\left(1+\frac{16 k^{2} S_{0}}{9 x}-\frac{4 k^{2}}{9} x^{2}\right)^{-1} d x^{2}
\end{aligned}
$$

Comparing eq. (67) with the well-known form of the Schwarzschild-de Sitter solution

$$
\begin{aligned}
d s^{2}= & -\left(1-\frac{2 m}{x}-\frac{8 \pi \rho_{\Lambda}}{3} x^{2}\right) d T^{2}+x^{2} d \Omega^{2} \\
& +\left(1-\frac{2 m}{x}-\frac{8 \pi \rho_{\Lambda}}{3} x^{2}\right)^{-1} d x^{2} ;
\end{aligned}
$$

one deduces the physical meaning of $S_{0}$,

$$
S_{0}=-\frac{9 m}{8 k^{2}} \text {. }
$$

Then, the Schwarzschild-de Sitter solution in comoving coordinates is given by

$$
\begin{aligned}
e^{\omega} & =\left(r^{3 / 2} e^{k t}-\frac{9 m}{8 k^{2}} r^{-3 / 2} e^{-k t}\right)^{4 / 3}, \\
e^{\bar{\omega}} & =e^{\omega} \omega^{\prime 2} / 4 .
\end{aligned}
$$

\section{B. $\Lambda$ CDM universe}

Eqs. (60) and (61) show that we recover the homogenous and isotropic, spatially flat universe with a mixture of dust matter and dark energy if and only if we set $P=-\frac{3 \rho_{0}}{16 k^{2}} r^{3 / 2}$. This solution is obtained for

$$
\begin{aligned}
e^{\omega} & =\left(r^{3 / 2} e^{k t}-\frac{3 \rho_{0}}{16 k^{2}} r^{3 / 2} e^{-k t}\right)^{4 / 3}, \\
e^{\bar{\omega}} & =e^{\omega} \omega^{\prime 2} / 4 .
\end{aligned}
$$

The density of dust/dark matter and the scale factor are given by

$$
\rho_{d}=\frac{\rho_{0}}{a^{3}}, \quad a=\left(e^{k t}-\frac{3 \rho_{0}}{16 k^{2}} e^{-k t}\right)^{2 / 3},
$$

where $t>\frac{1}{2 k} \ln \frac{3 \rho_{0}}{16 k^{2}}$, and where $t=\frac{1}{2 k} \ln \frac{3 \rho_{0}}{16 k^{2}}$ represents the Big Bang singularity.

\section{Black hole in a $\Lambda$ CDM universe}

The inspection of eqs. (70) and (71) suggests that the solution for a black hole in a $\Lambda$ CDM universe should be given by

$$
\begin{aligned}
e^{\omega} & =\left(r^{3 / 2} e^{k t}-\frac{9 m}{8 k^{2}} r^{-3 / 2} e^{-k t}-\frac{3 \rho_{0}}{16 k^{2}} r^{3 / 2} e^{-k t}\right)^{4 / 3}, \\
e^{\bar{\omega}} & =e^{\omega} \omega^{\prime 2} / 4 .
\end{aligned}
$$

It is clear, by comparison of eqs. (73) and (60), that this is indeed a solution. The substitution of eq. (73) into the Einstein equations gives the density of dust

$$
\rho_{d}=\frac{\rho_{0}\left(32 r^{3} e^{k t}+6 m e^{-k t}-\rho_{0} r^{3} e^{-k t}\right)^{2}}{R^{3}\left(32 r^{3} e^{k t}-6 m e^{-k t}-\rho_{0} r^{3} e^{-k t}\right)^{2}} .
$$


This quantity is positive on the entire spacetime manifold. If $m=0$, we have $\rho_{d}=\rho_{0} / R^{3}$, the usual scaling.

\section{BLACK HOLE EVOLUTION IN A $\Lambda$ CDM UNIVERSE}

Here we investigate the evolution of a black hole $\mathrm{AH}$ in a $\Lambda \mathrm{CDM}$ universe. For this purpose, we rewrite the metric in Schwarzschild coordinates using

$$
x \equiv e^{-2 k t / 3} r\left(e^{2 k t}-\frac{9 m}{8 k^{2} r^{3}}-\frac{3 \rho_{0}}{16 k^{2}}\right)^{2 / 3},
$$

eq. (53) becomes

$$
\begin{aligned}
d s^{2}= & -\left(1-\frac{2 m}{x}-\frac{4 k^{2}}{9} x^{2}-\frac{\rho_{0}}{3 x} r^{3}\right) d t^{2} \\
& -2\left(\frac{2 m}{x}+\frac{4 k^{2}}{9} x^{2}+\frac{\rho_{0}}{3 x} r^{3}\right)^{1 / 2} d t d x \\
& +d x^{2}+x^{2} d \Omega^{2} .
\end{aligned}
$$

Introducing the new time variable $T$ with

$$
\begin{aligned}
d T= & J^{-1} d t+J^{-1}\left(1-\frac{2 m}{x}-\frac{4 k^{2}}{9} x^{2}-\frac{\rho_{0}}{3 x} r^{3}\right)^{-1} \\
& \cdot\left(\frac{2 m}{x}+\frac{4 k^{2}}{9} x^{2}+\frac{\rho_{0}}{3 x} r^{3}\right)^{1 / 2} d x
\end{aligned}
$$

where $J(t, x)$ is an integrating factor, we obtain a black hole solution in a $\Lambda$ CDM universe.

$$
\begin{aligned}
d s^{2}= & -\left(1-\frac{2 m}{x}-\frac{4 k^{2}}{9} x^{2}-\frac{\rho_{0}}{3 x} r^{3}\right) J^{2} d T^{2}+x^{2} d \Omega^{2} \\
& +\left(1-\frac{2 m}{x}-\frac{4 k^{2}}{9} x^{2}-\frac{\rho_{0}}{3 x} r^{3}\right)^{-1} d x^{2}
\end{aligned}
$$

The equation of the AHs is

$$
g_{00}=1-\frac{2 m}{x}-\frac{4 k^{2}}{9} x^{2}-\frac{\rho_{0}}{3 x} r^{3}=0,
$$

so

$$
r=\left(\frac{3 x}{\rho_{0}}-\frac{6 m}{\rho_{0}}-\frac{4 k^{2} x^{3}}{3 \rho_{0}}\right)^{1 / 3} .
$$

The substitution of eq. (80) into eq. (75) yields the equation of the AHs, using which the AHs evolution is plotted in Fig. 3. The black hole AH shrinks while the cosmic AH expands with the expansion of the universe. There was a time in the past at which the two horizons coincided and before which no horizon existed and the singularity was naked. Late in the history of the universe, both black hole and cosmic AHs approach constant values.

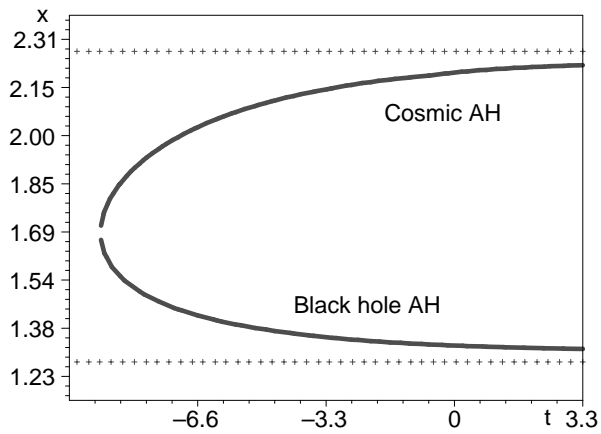

FIG. 3: In a $\Lambda$ CDM universe, the black hole AH (lower curve) shrinks while the cosmic AH (upper curve) expands with the cosmic expansion. There was a time at which the two horizons coincided and before which no horizon existed and the singularity was naked. Late in the history of the universe, both black hole and cosmic AHs approach constant size. The plots correspond to the parameter values $m=2.2 \cdot 10^{22} M_{\odot}, \rho_{0}=0.27 \rho_{C}, \rho_{\Lambda}=0.73 \rho_{C} . \quad \rho_{C}$ is the current cosmic density. The unit of $t$ is the present Hubble time $H_{0}^{-1}$.

\section{BLACK HOLE IN A QUINTOM UNIVERSE}

The first year WMAP data combined with the $2 \mathrm{dF}$ galaxy survey and the supernova Ia data favor the phantom energy equation of state of the cosmic fluid $w<$ -1 over the cosmological constant $(w=-1)$ and the quintessence field $(w>-1)[60,61]$. The data seem to slightly favor an evolving dark energy with $w<-1$ at the present epoch and $w>-1$ in the near past [62]. The dark energy candidate with evolving $w$ is named quintom [32]. In this section, we look for the solution for a black hole in a quintom-dominated universe. We take the energy-momentum tensor for the quintom fluid to be of the form

$$
T_{\mu \nu}=(\rho+p) U_{\mu} U_{\nu}+p g_{\mu \nu},
$$

and the line element in the form of eq. (53). In comoving coordinates, the four-velocity is $U^{\mu}=(1,0,0,0)$ and the Einstein equations are

$$
\begin{aligned}
& G_{0}^{0}=8 \pi \rho, \quad G_{1}^{0}=0, \\
& G_{1}^{1}=-8 \pi p, \quad G_{2}^{2}=-8 \pi p .
\end{aligned}
$$

From $G_{1}^{0}=0$ (again, there is no accretion), we obtain

$$
e^{\bar{\omega}}=e^{\omega} \omega^{\prime 2} / 4 \text {. }
$$

Again, we set the integration "constant" to unity and, inserting eq. (83) into eqs. (82), we obtain

$$
\ddot{\omega}^{\prime}+\frac{3}{2} \dot{\omega} \dot{\omega}^{\prime}=0
$$

and

$$
\ddot{\omega}+\frac{3}{4} \dot{\omega}^{2}=-8 \pi K(t),
$$


where $K(t)$ is an integration "constant". This equation is consistent with $G_{1}^{1}=-8 \pi p$ only if the pressure satisfies

$$
p=K(t)
$$

i.e., it is spatially homogenous, which can be seen as the consequence of three facts. First, we have set $f(R)=1$ for a spatially flat background. Second, we use comoving coordinates and, third and most important, we have taken the source to be a single perfect fluid. Therefore, the comoving observer will see a homogenous pressure. The Einstein equations simplify to

$$
\begin{aligned}
\ddot{\omega}+\frac{3}{4} \dot{\omega}^{2} & =-8 \pi p(t), \\
\frac{\dot{\omega}^{\prime} \dot{\omega}}{\omega^{\prime}}+\frac{3}{4} \dot{\omega}^{2} & =8 \pi \rho(t, r) .
\end{aligned}
$$

Now we have three functions $\omega, p$, and $\rho$ but only two equations and the system is not closed. For simplicity, we assign the pressure in the form

$$
p=-\frac{p_{0}}{\left(t_{0}-t\right)^{2}}
$$

where $p_{0}$ is a positive constant to keep the pressure always negative, and $t_{0}$ is a positive constant identifying the Big Rip singularity. The general solution of eqs. (87) is then given by

$$
\begin{aligned}
e^{\omega}= & {\left[P(r)\left(t_{0}-t\right)^{\frac{1}{2}\left(1-\sqrt{1+24 \pi p_{0}}\right)}\right.} \\
& \left.+S(r)\left(t_{0}-t\right)^{\frac{1}{2}\left(1+\sqrt{1+24 \pi p_{0}}\right)}\right]^{4 / 3},
\end{aligned}
$$

where $S$ and $P$ are arbitrary functions of $r$. It is convenient to choose

$$
P=r^{3 / 2}
$$

then the candidate solution for a black hole in a quintom universe is

$$
\begin{aligned}
e^{\omega}= & {\left[r^{3 / 2}\left(t_{0}-t\right)^{\frac{1}{2}\left(1-\sqrt{1+24 \pi p_{0}}\right)}\right.} \\
& -\frac{3}{2} \sqrt{2 m}\left(t_{0}-t\right)^{\frac{1}{2}\left(1+\sqrt{1+24 \pi p_{0}}\right)} \\
& \left.-\frac{3}{2} \sqrt{\frac{8 \pi \rho_{0}}{3}} r^{3 / 2}\left(t_{0}-t\right)^{\frac{1}{2}\left(1+\sqrt{1+24 \pi p_{0}}\right)}\right]^{4 / 3}
\end{aligned}
$$

If $m=0$, we get a quintom-dominated cosmology while, if $p_{0}=0$, we get a candidate solution for a black hole in a matter-dominated universe. Substituting eq. (91) into eqs. (87), we find that the energy density is positive and inhomogeneous.

\section{EVOLUTION OF A BLACK HOLE IN A QUINTOM UNIVERSE}

To investigate the evolution of the black hole $\mathrm{AH}$, we rewrite the metric in Schwarzschild coordinates using

$$
\begin{aligned}
x \equiv[ & {\left[r^{3 / 2}\left(t_{0}-t\right)^{\frac{1}{2}\left(1-\sqrt{1+24 \pi p_{0}}\right)}\right.} \\
& -\frac{3}{2} \sqrt{2 m}\left(t_{0}-t\right)^{\frac{1}{2}\left(1+\sqrt{1+24 \pi p_{0}}\right)} \\
& \left.-\frac{3}{2} \sqrt{\frac{8 \pi \rho_{0}}{3}} r^{3 / 2}\left(t_{0}-t\right)^{\frac{1}{2}\left(1+\sqrt{1+24 \pi p_{0}}\right)}\right]^{2 / 3}
\end{aligned}
$$

and define $k \equiv \sqrt{1+24 \pi p_{0}}$. Eq. (53) becomes

$$
\begin{aligned}
d s^{2} & =-\left(1-\mathscr{L}^{2}\right) d t^{2}+d x^{2}-2 \mathscr{L} d t d x+x^{2} d \Omega^{2} \\
\mathscr{L} & \equiv \frac{\partial x}{\partial t} .
\end{aligned}
$$

Introducing the time coordinate

$$
d T=J^{-1}\left(d t+\frac{\mathscr{L}}{1-\mathscr{L}^{2}} d x\right)
$$

where $J$ is an integrating factor, the metric becomes

$$
d s^{2}=-\left(1-\mathscr{L}^{2}\right) J^{2} d t^{2}+\frac{1}{1-\mathscr{L}^{2}} d x^{2}+x^{2} d \Omega^{2}(95)
$$

and the AHs are identified by

$$
1-\mathscr{L}^{2}=0
$$

Eq. (92) then yields

$$
r^{3 / 2}=\frac{1}{2} \frac{2 x^{3 / 2}+3 \sqrt{2 m}\left(t_{0}-t\right)^{\frac{1}{2}(1+k)}}{\left(t_{0}-t\right)^{\frac{1}{2}(1-k)}-\sqrt{6 \pi \rho_{0}}\left(t_{0}-t\right)^{\frac{1}{2}(1+k)}}
$$

Substituting eq. (97) into eq. (96), an explicit equation for the AHs in terms of $t$ and $x$ is obtained. The evolution of the AHs is reported in Fig. 4 . In the quintomdominated universe, the size of the black hole $\mathrm{AH}$ first decreases in the matter era and then increases during the phantom-dominated epoch [63]. The size of the cosmic $\mathrm{AH}$, instead, first increases and then decreases. There exists an instant of time in the past at which the two horizons coincided. Both horizons disappear before the Big Rip, leaving behind a naked singularity and violating Cosmic Censorship [37]. This result is consistent with our previous discussion in 21]. The naked singularity does not arise from regular Cauchy data, in fact it was already present before the appearance of the two apparent horizons.

The reason for the increase of the black hole mass in the phantom-dominated epoch can be understood as follows: the density of the universe is increasing, which makes the black hole $\mathrm{AH}$ also increase. Hence, the black hole mass (one half of the $\mathrm{AH}$ radius) increases. On the contrary, 


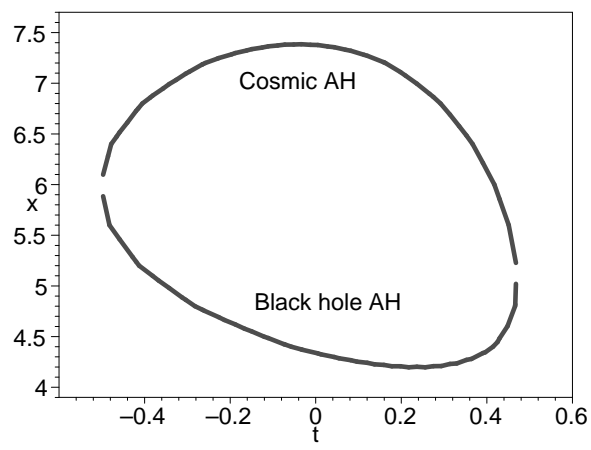

FIG. 4: In a quintom-dominated universe, the black hole $\mathrm{AH}$ (lower curve) first shrinks and then expands, while the cosmic AH (upper curve) first expands and then shrinks. There exist two instants of time, one in the past and one in the future, at which the two horizons coincide. Before or after these critical instants, no AH exists and a naked singularity is present. The plots correspond to the parameter values $m=1, \rho_{0}=$ $0.0002, p_{0}=0.001$, and $t_{0}=1$.

in a matter-dominated universe, the cosmic density is decreasing, so the black hole $\mathrm{AH}$ and the black hole mass decrease. In a cosmological constant-dominated universe, the cosmic density is constant and the black hole mass also stays constant.

\section{DISCUSSION AND CONCLUSIONS}

We have shown that several well-known and less-known solutions of the Einstein equations can not describe a black hole embedded in a matter-dominated universe sourced by a single perfect fluid. This includes the McVittie, Thakurta, Sultana-Dyer, Vaidya, and FaraoniJacques solutions. Motivated by this fact, we have constructed exact solutions of the Einstein equations purporting to represent a black hole embedded in a background universe which are as simple as possible, beginning with solutions with a single perfect fluid. These new solutions generalize the Lemaître-Tolman-Bondi metrics (which are restricted to dust universes) and do not exhibit accretion of the surrounding cosmic fluid onto the central black hole. We have found metrics which presumably describe a black hole immersed in a matterdominated, a matter plus dark energy-dominated universe, and a quintom-dominated universe, respectively.

The AH and the Misner-Sharp mass are related to the thermodynamics of dynamical black holes and FRW universes 42 45, 49 56, and, therefore, we investigate the evolution of the black hole and cosmic AHs, as well as the Misner-Sharp mass. We find that the black hole mass decreases in a matter- or matter plus cosmological constantdominated universe. In a quintom-dominated universe, the black hole mass decreases in the matter era, while it increases in the phantom-dominated epoch. The physical reason is that the cosmic density first decreases and then increases, which makes the radius of the black hole AH first decreasing and then increasing. Then, also the black hole mass (one half of the $\mathrm{AH}$ radius) first decreases and then increases. An interesting result is that the AHs will disappear and the singularity will become naked before the Big Rip is reached, in violation of the Cosmic Censorship Conjecture [37]. If the latter is correct, then phantom matter may not be allowed to exist in nature. This statement, however, must be taken with a grain of salt: in fact, it is based on a particular solution of the field equations which may be very special. While the result is still interesting because very few exact solutions are known to represent the physical situation under study, the solution proposed may still be too special, or finetuned, to draw general conclusions. The phenomenology reported here, however, matches that obtained with very different classes of solutions (which are indeed accreting cosmic fluid) in [21].

Another rather surprising result is that the black hole mass decreases in a matter-dominated universe. As the most strongly bound gravitational system, it seems intuitive that a black hole will have its mass increasing because of the swallowing of surrounding cosmic matter. Our solution represents cases in which there is no accretion onto the central black hole (as described by $G_{1}^{0}=0$ ) and in which the cosmic expansion "wins" over the local gravitational attraction. The universe is always expanding and the density of the cosmic fluid always decreases with the cosmic time. The seemingly bizarre behavior of the MS mass derives from the fact that it is really a mass sum (i.e., the mass of the background fluid is included), and it coincides with the Hawking-Hayward quasi-local mass [21]. In the absence of accretion of cosmic fluid onto the black hole, this mass sum decreases in any universe in which the cosmological fluid satisfies the weak energy condition and its energy density decreases with the cosmic expansion. In the presence of phantom matter (such as in the late epoch of a quintom-dominated universe), the weak energy condition is violated, the phantom energy becomes more concentrated, instead of being diluted, with the cosmic expansion, and the mass sum increases.

Future work will concentrate on studying the generality of the solutions presented here, and of previous solutions interpreted as black holes embedded in a cosmological space. To fully support the interpretation of these solutions as black holes in a cosmological background, it is important that a detailed investigation on the global structure of these spacetimes be provided. Until this global analysis is available, we cannot yet claim that our solutions do represent black holes embedded in expanding universes, although there is circumstantial evidence. This analysis will be carried out in future publications. 


\section{ACKNOWLEDGMENTS}

We thank J. D. Barrow, A. Krasinski and J. Taghizade for helpful communications. We also thank an anonymous referee for expert and insightful comments, which led to improvements in this manuscript. This work is supported by the National Science Foundation of China under the Key Project Grant 10533010, Grant 10575004, Grant 10973014, and the 973 Project (No.
2010CB833004). V.F. acknowledges the Natural Sciences and Engineering Research Council of Canada (NSERC).

After completion of this work, we learned that the solutions of Secs. V and VI can also be obtained as special cases of solutions studied by Krasinski, Hellaby, and Jacewicz (see Ref. [3]). The solution of Secs. IX and $\mathrm{X}$ was already contained in Ref. [64] and is analogous to class-II Szekeres models. We thank A. Krasinski for pointing this out.
[1] W.B. Bonnor, Class. Quantum Grav. 16, 1313 (1999).

[2] B.C. Nolan, Phys. Rev. D 58, 064006 (1998).

[3] J. Pachner, Phys. Rev. 132, 1837 (1963); Phys. Rev. B 137, 1379 (1965); W.M. Irvine, Ann. Phys. 32, 322 (1965); R.H. Dicke and P.J.E. Peebles, Phys. Rev. Lett. 12, 435 (1964); C. Callan, R.H. Dicke, and P.J.E. Peebles, Am. J. Phys. 33, 105 (1965); P. D'Eath, Phys. Rev. D 11, 1387 (1975); R.P.A. Newman and G.C. McVittie, Gen. Rel. Grav. 14, 591 (1982); R. Gautreau, Phys. Rev. D 29, 198 (1984); P.A. Hogan, Astrophys. J. 360, 315 (1990); J.L. Anderson, Phys. Rev. Lett. 75, 3602 (1995); W.B. Bonnor, Mon. Not. R. Astr. Soc. 282, 1467 (1996); A. Feinstein, J. Ibanez, and R. Lazkoz, Astrophys. J. 495, 131 (1998); K.R. Nayak, M.A.H. MacCallum, and C.V. Vishveshwara, Phys. Rev. D 63, 024020 (2000); V. Guruprasad, gr-qc/0005090, gr-qc/0005014 G.A. Baker Jr., astro-ph/0003152 A. Dominguez and J. Gaite, Europhys. Lett. 55, 458 (2001); T.M. Davis and C.H. Lineweaver, AIPC 555, 348 (2001); G.F.R. Ellis, Int. J. Mod. Phys. A 17, 2667 (2002); C. Stornaiolo, Gen. Rel. Grav. 34, 2089 (2002); T.M. Davis, C.H. Lineweaver, and J.K. Webb, Am. J. Phys. 71, 358 (2003); L. Lindegren and D. Dravins, Astron. Astrophys. 401, 1185 (2003); C.J. Gao, Class. Quantum Grav. 21, 4805 (2004); T.M. Davis and C.H. Lineweaver, Publ. Astr. Soc. Pac. 21, 97 (2004); T.M. Davis, PhD thesis, Univ. of New South Wales (astro-ph/0402278); D.P. Sheehan and V.G. Kriss, astro-ph/0411299, W.J. Clavering, Am.J.Phys. 74, 745 (2006); Z.-H. Li and A. Wang, Mod. Phys. Lett. A 22, 1663 (2007); O. Gron and O. Elgaroy, astro-ph/0603162 L.A. Barnes, M.J. Francis, J.B. James, and G.F. Lewis, Mon. Not. R. Astr. Soc. 373, 382 (2006); R. Lieu and D.A. Gregory, astro-ph/0605611 P.K.F. Kuhfittig, Int. J. Pure Appl. Math. 49, 577 (2008) gr-qc/0608120; G.S. Adkins, J. McDon- nell, and R.N. Fell, Phys. Rev. D 75, 064011 (2007); D.L. Wiltshire, New J. Phys. 9, 377 (2007); M. Sereno and P. Jetzer, Phys. Rev. D 75, 064031 (2007); J. D. Barrow and J. Stein-Schabes, Phys. Lett. A 103,315(1984); D. J. Shaw and J. D. Barrow, Phys. Rev. D 73, 123505 (2006); Phys. Rev. D 73, 123506 (2006); J. T. Firouzjaee and R. Mansouri, Gen. Relativ. Gravit. 42, 2431 (2010); A. Krasinski, Ch. Hellaby, Phys. Rev. D 69, 043502 (2004); P. Jacewicz and A. Krasinski, arXiv 1101.5307; D. A. Szafron and J. Wainwright, J. Math. Phys. 18, 1668(1977).

[4] P.D. Noerdlinger and V. Petrosian, Astrophys. J. 168, 1 (1971).

[5] H. Sato and K. Maeda, Prog. Theor. Phys. 70, 119 (1983).
[6] R. Sussman, Gen. Rel. Grav. 17, 251 (1985).

[7] P.D. D'Eath, Phys. Rev. D 11, 1387 (1975).

[8] M. Ferraris, M. Francaviglia, and A. Spallicci, Nuovo Cimento 111B, 1031 (1996).

[9] F.I. Cooperstock, V. Faraoni, and D.N. Vollick, Astrophys. J. 503, 61 (1998).

[10] B. Bolen, L. Bombelli, and R. Puzio, Class. Quantum Grav. 18, 1173 (2001).

[11] B.C. Nolan, Class. Quantum Grav. 16, 1227 (1999).

[12] B.C. Nolan, Class. Quantum Grav. 16, 3183 (1999).

[13] M.T. Busha, F.C. Adams, R.H. Wechsler, and A.E. Evrard, Astrophys. J. 596, 713 (2003).

[14] C.J. Gao and S.N. Zhang, Phys. Lett. B 595, 28 (2004).

[15] J. Sultana and C.C. Dyer, Gen. Rel. Grav. 37, 1349 (2005)

[16] M. Mizony and M. Lachièze-Rey, Astron. Astrophys. 434, 45 (2005); D. Izzo and A. Rathke, astro-ph/0504634 F.J. Oliveira, gr-qc/0610029 M. Lachièze-Rey, Class. Quantum Grav. 24, 2735 (2007); J. Rosales and J. Sanchez-Gomez, gr-qc/99810085; M. Carrera and D. Giulini, gr-qc/0602098 Class. Quantum Grav. 23, 7483 (2006); C. Lammerzhal and O. Preuss, gr-qc/0604052 S.G. Turyshev and J.G. Williams, Int. J. Mod. Phys. D 16, 2165 (2007); H.-J. Fahr and M. Siewert, gr-qc/0610034; J.G. Williams, S.G. Turyshev, and D.H. Boggs, Phys. Rev. Lett. 98, 059002 (2007); Y.V. Dumin, gr-qc/0610035.

[17] S. Nesseris and L. Perivolaropoulos, Phys. Rev. D 70, 123529 (2004).

[18] R.H. Price, arXiv:gr-qc/0508052

[19] A. Balaguera-Antolinez and M. Nowakowski, Class. Quantum Grav. 24, 2677 (2007).

[20] B. Mashhoon, N. Mobed, and D. Singh, Class. Quantum Grav. 24, 5031 (2007).

[21] C. Gao, X. Chen, V. Faraoni and Y. G. Shen, Phys. Rev. D 78, 024008 (2008).

[22] A. Ashtekar and B. Krishnan, Phys. Rev. Lett. 89, 261101 (2002).

[23] A. Krasinski, Inhomogeneous Cosmological Models (CUP, Cambridge, 1997).

[24] J.M. Senovilla, M. Mars, and R. Vera, Phys. World, July 1999, 20 (1999); W.B. Bonnor, Gen. Rel. Grav. 38, 1005 (2000).

[25] M. Carrera and D. Giulini, Rev. Mod. Phys. 82, 169 (2010).

[26] G. C. McVittie, Mon. Not. R. Astron. Soc. 93, 325 (1933).

[27] M.L. McClure and C.C. Dyer, Class. Quantum Grav. 23, 1971 (2006); Gen. Rel. Gravit. 38, 1347 (2006). 
[28] A. Einstein and E.G. Straus, Rev. Mod. Phys. 17, 120 (1945); 18, 148 (1946).

[29] P. C. Vaidya, Pramana 8, 51 (1977).

[30] S.N.G. Thakurta, Indian J. Phys. 55B, 304 (1981).

[31] V. Faraoni and A. Jacques, Phys. Rev. D 76, 063510 (2007).

[32] B. Feng, X.L. Wang and X.M. Zhang, Phys. Lett. B 607, 35 (2005); B. Feng, M. Li, Y.S. Piao and X.M. Zhang, Phys. Lett. B 634, 101 (2006); Z.K. Guo, Y.S. Piao, X.M. Zhang and Y.Z. Zhang, Phys. Lett. B 608, 177 (2005); X. Zhang, Commun. Theor. Phys. 44, 762 (2005); X.F. Zhang, H. Li, Y.S. Piao and X.M. Zhang, Mod. Phys. Lett. A 21, 231 (2006); H. Wei, R.G. Cai and D.F. Zeng, Class. Quantum Grav. 22, 3189 (2005); M.Z. Li, B. Feng and X.M. Zhang, J. Cosmol. Astropart. Phys. 0512, 002 (2005); A. Anisimov, E. Babichev and A. Vikman, J. Cosmol. Astropart. Phys. 0506, 006 (2005); H. Wei and R.G. Cai, Phys. Lett. B 634, 9 (2006); H. Wei and R.G. Cai, Phys. Rev. D 73, 083002 (2006); Z.K. Guo, Y.S. Piao, X. Zhang and Y.Z. Zhang, Phys. Rev. D 74, 127304 (2006); Y.F. Cai, H. Li, Y.S. Piao and X.M. Zhang, Phys. Lett. B 646, 141 (2007)

[33] Y.B. Zeldovich and I.D. Novikov, Sov. Astr. A.J. 10, 602 (1967); S.W. Hawking, Phys. Rev. Lett. 26, 1344 (1971); Mon. Not. R. Astr. Soc. 152, 75 (1971); B.J. Carr and S.W. Hawking, Mon. Not. R. Astr. Soc. 168, 399 (1974); N. Sakai and J.D. Barrow, Class. Quantum Grav. 18, 4717 (2001).

[34] T. Harada and B.J. Carr, Phys. Rev. D 71, 104009 (2005); 71, 104010 (2005); 72, 044021 (2005); T. Harada, H. Maeda, and B.J. Carr, Phys. Rev. D 74, 024024 (2006); B.J. Carr, astro-ph/0511743

[35] H. Saida, T. Harada, and H. Maeda, Class. Quantum Grav. 24, 4711 (2007).

[36] S.W. Hawking, Phys. Rev. Lett. 26, 1344 (1971); J.D. Bekenstein, Phys. Rev. D 7, 2333 (1973); Phys. Rev. D 9, 3292 (1974); J.M. Bardeen, B. Carter and S.W. Hawking, Commun. Math. Phys. 31, 161 (1973); S.W. Hawking, Phys. Rev. D 13, 191 (1976); W. Israel, Phys. Rev. Lett. 57, 397 (1986); V. Iyer and R.M. Wald, Phys. Rev. D 50, 846 (1994).

[37] R. Penrose, Riv. Nuovo Cim. 1, 252 (1969) [Gen. Rel. Grav. 34, 1141 (2002)].

[38] H. Bondi, Mon. Not. R. Astr. Soc. 107, 410 (1947).

[39] M.N. Célérier, Astron. Astrophys. 353, 63 (2000).

[40] R.C. Tolman, Proc. Nat. Acad. Sci. 20, 3 (1934).

[41] J.R. Oppenheimer and H. Snyder, Phys. Rev. D 56, 455 (1939).

[42] S.A. Hayward, S. Mukohyama, and M.C. Ashworth, Phys. Lett. A 256, 347 (1999); S.A. Hayward, Class. Quantum Grav. 15, 3147 (1998).

[43] D. Bak and S.J. Rey, Class. Quantum Grav. 17, L83 (2000).

[44] R.G. Cai and S.P. Kim, J. High Energy Phys. 02, 050 (2005).

[45] X. Zhang, Eur. Phys. J. C 60, 661 (2009); D. Brill, G. Horowitz, D. Kastor, J. Traschen, Phys. Rev. D 49 , 840 (1994); H. Saida, T. Harada, and H. Maeda, Class. Quantum Grav. 24, 4711 (2007); M. Nozawa and H. Maeda, Class. Quantum Grav. 25, 055009 (2008); A. Ashtekar and G.J. Galloway, Adv. Theor. Math. Phys. 9, 1 (2005) arXiv:gr-qc/0503109; A. Ashtekar and B. Krishnan, Living Rev. Rel. 7, 10 (2004); Phys. Rev. D 68, 104030 (2003); Phys. Rev. Lett. 89, 261101
(2006); A. Ashtekar and A. Corichi, Class. Quantum Grav. 17, 1317 (2000); A. Ashtekar, A. Corichi, and D. Sudarsky, Class. Quantum Grav. 20, 3413 (2003); A. Ashtekar, J. Engle, T. Pawlowski and C. Van Den Broeck, Class. Quantum Grav. 21, 2549 (2004); A. Ashtekar, arXiv:gr-qc/0306115; A. Ashtekar, C. Beetle, and J. Lewandowski, Class. Quantum Grav. 19, 1195 (2002); Phys. Rev. D 64, 044016 (2001); A. Ashtekar, C. Beetle, O. Dreyer, S. Fairhurst, B. Krishnan, J. Lewandowski, and J. Wisniewski, Phys. Rev. Lett. 85, 3564 (2000); A. Ashtekar, S. Fairhurst and B. Krishnan, Phys. Rev. D 62, 104025 (2000); A. Ashtekar, C. Beetle and S. Fairhurst, Class. Quantum Grav. 17, 253 (2000); 16, L1 (1999); A.B. Nielsen and M. Visser, Class. Quantum Grav. 23, 4637 (2006); D. Kotawala, S. Sarkar, and T. Padmanabhan, Phys. Lett. B 652, 338 (2007); M. Nadalini, L. Vanzo, and S. Zerbini, Phys. Rev. D 77, 024047 (2008); M.L. McClure, arXiv:0709.3288 J.A. de Freitas Pacheco and J.E. Horvath, Class. Quantum Grav. 24, 5427 (2007); D.C. Guariento, J.E. Horvath, P.S. Custodio, and J.A. de Freitas Pacheco, Gen. Rel. Grav. 40, 1593 (2008); P.S. Custodio and J.E. Horvath, Int. J. Mod. Phys. D 14, 257 (2005); I. Booth, Can. J. Phys. 83, 1073 (2005); Z.-H. Li and A. Wang, Mod. Phys. Lett. A 22, 1663 (2007); G. Izquierdo and D. Pavon, Phys. Lett. B 639, 1 (2006); S. Chen and J. Jing, Class. Quantum Grav. 22, 4651 (2005); T. Clifton, D.F. Mota, and J.D. Barrow, Mon. Not. R. Astr. Soc. 358, 601 (2005); N. Sakai and J.D. Barrow, Class. Quantum Grav. 18, 4717 (2001); S.A. Hayward, Phys. Rev. D 70, 104027 (2004); Phys. Rev. Lett. 93, 251101 (2004); arXiv:gr-qc/0008071 Phys. Rev. Lett. 81, 4557 (1998); Phys. Rev. D 53, 1938 (1996); Class. Quantum Grav. 11, 3025 (1994); Phys. Rev. D 49, 6467 (1994); Phys. Rev. D 49, 6467 (1994); S. Mukohyama and S.A. Hayward, Class. Quantum Grav. 17, 2153 (2000); H. Maeda, arXiv:0704.2731 G. Kang, Phys. Rev. D 54, 7483 (1996); T. Jacobson and G. Kang, Class. Quantum Grav. 10, L201 (1993); C.C. Dyer and E. Honig, J. Math. Phys. 20, 409 (1979); S. Nojiri and S.D. Odintsov, Phys. Rev. D. 70, 103522 (2004).

[46] N. Kaloper, M. Kleban and D. Martin, Phys. Rev. D 81, 104044 (2010).

[47] K. Lake and M. Abdelqader, Phys. Rev. D 84, 044045 (2011).

[48] R. Nandra, A.N. Lasenby, and M.P. Hobson, arXiv:1104.4447, arXiv:1104.4458.

[49] Y. Gong and A. Wang, Phys. Rev. Lett. 99, 211301 (2007).

[50] M. Akbar and R.G. Cai, Phys. Rev. D 75, 084003 (2007).

[51] R.G. Cai and L.M. Cao, Phys. Rev. D 75, 064008 (2007).

[52] R.G. Cai and L.M. Cao, Nucl. Phys. B 785, 135 (2007).

[53] M. Akbar and R.G. Cai, Phys. Lett. B 648, 243 (2007).

[54] A. Sheykhi, B. Wang and R.G. Cai, Phys. Rev. D 76, 023515 (2007).

[55] A. Sheykhi, B. Wang and R.G. Cai, Nucl. Phys. B 779, 1 (2007).

[56] R. Di Criscienzo, M. Nadalini, L. Vanzo, S. Zerbini, and G. Zoccatelli, Phys. Lett. B 657, 107 (2007).

[57] C.M. Misner and D.H. Sharp, Phys. Rev. 136, 571 (1964).

[58] S. Perlmutter et al., Astrophys. J. 517, 565 (1999).

[59] A.G. Riess et al., Astron. J. 116, 1009 (1998).

[60] P.S. Corasaniti, M. Kunz, D. Parkinson, E.J. Copeland and B.A. Bassett, Phys. Rev. D 70, 083006 (2004). 
[61] U. Alam, V. Sahni, T.D. Saini and A.A. Starobinsky, Mon. Not. Roy. Astron. Soc. 354, 275 (2004).

[62] B. Feng, X.L. Wang and X.M. Zhang, Phys. Lett. B 607, 35 (2005).
[63] R.R. Caldwell, M. Kamionkowski, and N.N. Weinberg, Phys. Rev. Lett. 91, 071301 (2003).

[64] D. A. Szafron, J. Math. Phys. 18, 1673(1977). 